\title{
Medizinische Call Center entlasten den ärztlichen Notfalldienst
}

\author{
A. Meer ${ }^{b}$, A. Wirthner ${ }^{a}$, C. Simonin ${ }^{b}$
}

\section{Einleitung}

Bereits in der Novemberausgabe des «Lancet» von 1879 wurde das Telefon als technisches Hilfsmittel in der medizinischen Grundversorgung beschrieben:

"The Yankees are rapidly finding out the benefits of the telephone. A newly made grandmamma, we are told, was recently awakened by the bell at midnight, and told by her inexperienced daughter, 'Baby has the croup. What shall I do with it?' Grandmamma replied she would call the family doctor, and would be there in a minute. Grandmamma woke the doctor, and told him the terrible news. He in turn asked to be put in telephonic communication with the anxious mamma. 'Lift the child to the telephone, and let me hear it cough,' he commands. The child is lifted and it coughs. 'That's not the croup' he declares, and declines to leave his house on such small matters. He advises grandmamma also to stay in bed; and all anxiety quieted, the trio settle down happy for the night." (Lancet 1879;Nov. 29)

Über Jahrzehnte wurde die Telephonie in der Medizin zur Übermittlung von administrativen, logistischen und medizinischen Daten und Informationen eingesetzt. Im Zuge der Weiterentwicklung der Informations- und Kommunikationstechnologie entstanden in den USA ab den siebziger Jahren Telefonzentralen mit informatikgestützter Anrufverteilung. Mit der Einführung von medizinischen Call Centern Anfang der neunziger Jahre erfolgte eine wesentliche Erweiterung und Verbesserung des Angebotes. Computerbasierte Informationssysteme unterstützen die nichtärztlichen medizinischen Fachpersonen und ermöglichen ihnen, qualitativ hochstehende Beratungs- und Informationsgespräche am Telefon zu führen. Die medizinische Beratung von Patienten mit akuten Gesundheitsbeschwerden wurde professionalisiert und mit medizinischen Expertensystemen hinterlegt. In Europa wurde die computerassistierte
Telefontriage zuerst in Grossbritannien und später in den Niederlanden eingeführt. Unter dem Namen NHS-Direct ist die Dienstleistung in England seit dem Jahre 2001 landesweit verfügbar. In der Schweiz wurde die computerassistierte Telefontriage im Frühjahr 2000 erstmals durch die Firma Medvantis eingeführt.

Die Dienstleistung in dieser Form war neu für das schweizerische Gesundheitswesen und konnte von Grund auf konzipiert werden, ohne dass historische «Altlasten» bewältigt werden mussten. Deshalb konnte in diesem Bereich eine relativ schnelle Informatisierung der Medizin erfolgen. Dies ist eine wichtige Grundvoraussetzung für die klare Prozessführung, die Dokumentation und für das Qualitätsmanagement medizinischer Abläufe.

In den letzten fünf Jahren haben sich die medizinischen Call-Center-Anbieter in der Schweiz etabliert und profiliert. Nicht nur Krankenversicherer, sondern auch Einzel- und Gruppenpraxen sowie ärztliche Notfalldienste und Hausarztnetzwerke beanspruchen deren Dienstleistungen.

\section{Die computerassistierte Telefontriage}

Ganz grundsätzlich steht bei medizinischen Triagen die symptombasierte Evaluation der Dringlichkeit der Beschwerden und die Festlegung weiterführender Massnahmen im Vordergrund. Die Diagnosestellung erfolgt erst sekundär mit den dafür angemessenen und bereits bestehenden personellen und infrastrukturellen Ressourcen unseres Gesundheitswesens.

Das computerbasierte medizinische Expertensystem führt die Gesundheitsberaterin in der symptomorientierten Befragung und Beurteilung. Die einzelnen Prozessschritte werden kontinuierlich elektronisch dokumentiert und strukturiert abgelegt (Abb. 1). Aufgrund des Fachwissens der Gesundheitsberaterinnen sowie der Entscheidungsunterstützung durch das Computersystem wird den Anrufenden eine der in Tabelle 1 aufgeführten grundsätzlichen Handlungsempfehlungen gegeben. Die Argumente, welche seitens des Expertensystems zu einer be- 
Tabelle 1

Triagestufen, Bezeichnungen und Handlungsempfehlungen.

\begin{tabular}{lll}
\hline Triagestufe & Bezeichnung & Handlungsempfehlung \\
\hline 1 & Sofort & $\begin{array}{l}\text { Vermittlung an den regionalen } \\
\text { Rettungsdienst 144 mit der } \\
\text { Empfehlung der notfallmässigen } \\
\text { stationären Weiterbetreuung }\end{array}$ \\
\hline 2 & Empfehlung zur sofortigen \\
& & $\begin{array}{l}\text { ambulanten Notfallkonsultation } \\
\text { durch den Hausarzt oder dessen } \\
\text { Vertretung }\end{array}$ \\
\hline 3 & Mässig dringend & $\begin{array}{l}\text { Empfehlung zur Konsultation } \\
\text { beim Hausarzt oder dessen } \\
\text { Vertretung innerhalb 24 Stunden }\end{array}$ \\
\hline 4 & Routinekonsultation & $\begin{array}{l}\text { Empfehlung zur Hausarztkonsul- } \\
\text { tation innerhalb 2-7 Tagen }\end{array}$ \\
\hline 5 & Selbstbehandlung & Keine Arztkonsultation. Die \\
& & $\begin{array}{l}\text { Gesundheitsberaterin erteilt } \\
\text { Ratschläge zur Selbstbehandlung }\end{array}$ \\
& &
\end{tabular}

Abbildung 1

Die Gesundheitsberaterin wird in der strukturierten symptomorientierten Befragung geführt.

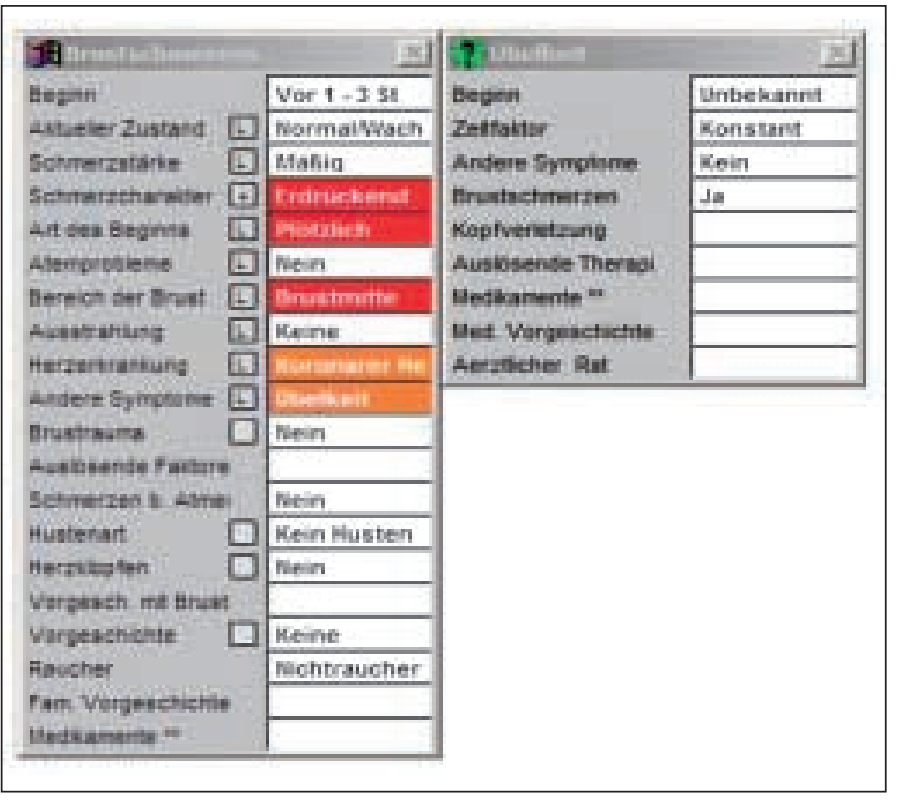

Abbildung 2

Die Argumente des Expertensystems zu einer bestimmten Empfehlung werden explizit ausformuliert.

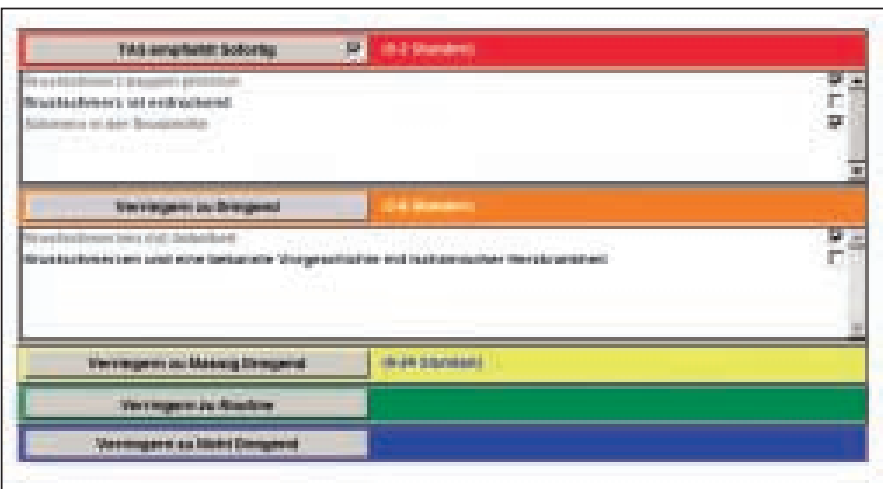

stimmten Empfehlung geführt haben, werden explizit formuliert und dokumentiert. Die Gesundheitsberaterin bestätigt durch die Aktivierung der entsprechenden Checkbox, dass sie die Expertenargumente in ihre Entscheidung einbezogen hat (Abb. 2).

Im medizinischen Call Center von Medvantis werden sämtliche Anrufe am Anfang («Konsultationsanlass», Sicht des Anrufenden) und am Ende des Gespräches («Gesundheitsproblem», Sicht der medizinischen Fachperson) ICPC-2kodiert. Die mehr als 100000 jährlichen, elektronischen Datensätze ergeben einen Fundus für die wissenschaftliche Forschung im Bereiche der medizinischen Grundversorgung und für die Versorgungsplanung.

\section{Evaluation der Dienstleistung}

Im Jahre 2004 wurde seitens der Cochrane Library eine Übersichtsarbeit präsentiert, welche die zahlreichen Resultate von repräsentativen Forschungsarbeiten in bezug auf den Effekt und die Patientenzufriedenheit der Telefontriage zusammenfasst [1]. Die Arbeit zeigt, dass durch den Einbezug der medizinischen Telefontriage die Arbeitslast der Grundversorger im Notfallbereich vermindert werden kann und dass aufgrund der computerassistierten Telefontriage mindestens $50 \%$ der Anrufe durch die Gesundheitsberaterinnen am Telefon erledigt werden konnten. Die Triagezentren, welche mit Pflegefachpersonen und computergestützten Expertensystemen arbeiten, standen den ärztlichen Dienstleistern bezüglich des Beratungsresultates in keiner Weise nach.

Gemäss einer Studie von Flaig et al. [2] an zwei Schweizer Spitälern (Kantonsspital Baden, Bezirksspital Brugg) hätten $80 \%$ der Patienten, welche sich mit Beschwerden direkt an der Notfallpforte des Spitals gemeldet haben, grundsätzlich in den Praxen der Grundversorger behandelt werden können. Die subjektive Dringlichkeit, die Abwesenheit des Hausarztes, die räumliche Nähe des Spitals und die Unkenntnis über den Notfalldienst wurden als wichtigste Gründe genannt, das Spital direkt aufzusuchen, ohne vorher den Haus- oder Notfallarzt zu kontaktieren.

Eine Studie zur medizinischen Telefontriage des Institutes für Sozial- und Präventivmedizin der Universität Bern bestätigte die obengenannte Beobachtung einer inadäquaten medizinischen Ressourcennutzung [3]. Lediglich 2\% der Anrufenden wurden von der Gesundheitsberaterin in ihrer Absicht bestärkt, direkt die Notfallstation des Spitals aufzusuchen. 81\% der 
Anrufenden mit dieser Absicht sind nach der Beratung von ihrem ursprünglichen Vorhaben abgewichen und haben sich selbst, vom Hausarzt oder dessen Vertretung behandeln können. Nebst der Arbeitslast der Hausärzte im ambulanten Notfalldienst kann durch die gezielte Einführung der computerassistierten Telefontriage auch die Fehlbeanspruchung der Notfallstationen der Spitäler verringert werden.

\section{Erfahrungsbericht der Praxis Bubenberg}

«Aber wir machen doch jetzt schon alles perfekt», war der Grundtenor unter den sieben Ärztinnen und Ärzten der Praxis Bubenberg bei der Diskussion über die Einführung einer professionellen Telefontriage ausserhalb der Praxisöffnungszeiten.

Dass eine 24-Stunden-Erreichbarkeit, nebst Hausbesuchen, die Grundvoraussetzung für eine seriöse Hausarztmedizin bildet, war seit der Praxisgründung vor bald 10 Jahren klar. Der praxiseigene Notfall- und Pikettdienst funktioniert zwar gut, trägt aber wie in jeder Grundversorgerpraxis durch die Störungen des Privatlebens, die ständige Konfrontation mit nicht immer zeit- und situationsgerechten Ansprüchen von Patientinnen und Patienten und die Beeinträchtigung des ungestörten Schlafs erheblich zum beruflichen Stress bei [4].

Die Überzeugung, dass die Patienten durch niemanden und zu keiner Zeit besser beraten werden als durch den persönlichen Hausarzt, geriet im Verlauf der Diskussion ins Wanken: Wie objektiv triagieren und beraten wir morgens um zwei Uhr, schlaftrunken und aus Träumen geholt, konfrontiert mit komplexen Situationen, emotional oft vorbelastet durch kräfteraubende Begleitungen und Betreuungen? Wie war doch die Geschichte unseres ansonsten an Besonnenheit und Geduld nicht zu übertreffenden Praxiskollegen, der sich, nächtens von einer Patientin geweckt, mit einem wutentbrannten Faustschlag gegen die Wand einen Bruch des Mittelhandknochens zuzog? War sein damaliger Triageentscheid richtig? Ist die Vorstellung und Anforderung mancher Ärzte an sich selbst, immer ausgeglichen und jedem Patienten gleich aufmerksam und freundlich zu begegnen, nicht naiv? Versuchen wir die Tatsache zu verleugnen, dass auch wir Ärzte ganz empfindlich und unterschiedlich auf die einzelnen Kranken und Situationen reagieren und dadurch Fehler begehen? Haben wir vielleicht Mühe mit dem Verzicht auf die Macht, erste und einzige Anlaufstelle zu sein? Ist dieser emotionale Widerstand so gross, dass nicht ein- mal wiederholtes Scheitern im Grundversorgeralltag ihn zu überwinden vermag? [5].

Die Praxis Bubenberg in Bern beschloss im Sommer 2002, die professionelle Telefontriage ausserhalb der Praxisöffnungszeiten einzuführen.

Ab Praxisschluss um 19 Uhr werden die einkommenden Telefonanrufe ans medizinische Call Center weitergeleitet. Gleichzeitig meldet die Praxisassistentin dort freie Sprechstundentermine für den folgenden Tag. Anrufende Patientinnen und Patienten werden vom medizinischen Call Center mit: «Praxis Bubenberg, grüessech» begrüsst und darauf hingewiesen, dass das Gespräch aus Sicherheitsgründen aufgezeichnet wird. Die Triagestufen 5 (Selbstbehandlung) und 4 (Routinekonsultation) werden von der Gesundheitsfachperson nach der computergestützten Befragung selbständig erledigt. Bei Triagestufe 3 (mässig dringend) wird für den folgenden Tag ein Sprechstundentermin vergeben. Eine Benachrichtigung des diensthabenden Praxisarztes oder gegebenenfalls der Sanitätsnotrufzentrale erfolgt ab der Stufe 2 (dringend) und 1 (sofort).

Bei Arbeitsbeginn um 7 Uhr druckt die Praxisassistentin die verschlüsselt übermittelten Dokumentationen der stattgefundenen Telefongespräche aus und übergibt sie an den jeweils zuständigen Arzt. Ebenso trägt sie die vergebenen Termine in der Agenda nach.

Die anfängliche Skepsis wich der Begeisterung. Etwa $40 \%$ der über 500 Anrufe ausserhalb der Praxisöffnungszeiten im Jahr 2004 konnten durch die Gesundheitsfachperson des medizinischen Call Centers selbständig erledigt werden. Das sind mehr als 200 Telefonanrufe, die der Dienstarzt nicht entgegenzunehmen hatte. Dieser Anteil liegt vermutlich darum etwas tiefer als die obenerwähnten $50 \%$, weil es sich um ein hausärztlich betreutes Kollektiv handelt.

Es hat uns gefreut, aber eigentlich nicht so sehr überrascht, dass aufgrund des Fachwissens der Gesundheitsfachperson und des computergestützten Pfades korrekte Triagen im Bereich der körperlichen Erkrankungen durchgeführt werden. Dass aber auch in komplexen, akuten oder chronifizierten psychosozialen Problemsituationen nahezu durchwegs korrekte Triagen erzielt wurden, war für uns sehr erstaunlich. Selbst die Beurteilung von fachlich und menschlich äusserst anspruchsvollen Patientinnen und Patienten, z.B. mit Angsterkrankungen, Suchtproblemen oder Borderlinestörungen, wurde korrekt durchgeführt und gab zu keiner Beanstandung Anlass. Die Akzeptanz der Beratung bei den Anrufenden ist gemäss unserer Erfahrung sehr hoch. 
Eine empathische Begleitung durch die menschliche Stimme am Telefon, gepaart mit dem computergestützten Wissen, scheint eine neue, bisher wenig genutzte Chance in der Gesundheitsversorgung zu bieten, die den Hausarzt bei seiner Alltagstätigkeit wirksam und qualitativ hochstehend entlasten kann. Dass dabei durch das Einschlagen eines adäquaten Pfades unnötige Kosten verhindert werden können, sei hier nur am Rande erwähnt.

\section{Strukturelle Probleme in der Notfalldienstorganisation}

Eine von der FMH unterstützte Publikation aus dem Jahre 2004 fasst eine grossangelegte Untersuchung zur Organisation des ambulanten ärztlichen Notfalldienstes in der Schweiz zusammen [6]. Die Autoren folgern, dass die heutigen Strukturen der Notfalldienstorganisationen vor allem in ländlichen Gebieten ineffizient und die Arbeitsleistung derselben nur ungenügend dokumentiert sind. Um längerfristig die gesetzlich vorgeschriebene notfallärztliche Versorgung der Bevölkerung sowohl in ländlichen Regionen wie auch in Städten und Agglomerationen der Schweiz sicherzustellen, ist eine Anpassung und Optimierung der Strukturen und Prozesse dringend erforderlich.

Die Autoren weisen darauf hin, dass die unbefriedigende Organisation des ambulanten Notfalldienstes ein Grund für eine beabsichtigte Praxisschliessung sein kann und dadurch längerfristig die Gefahr einer ärztlichen Unterversorgung in ländlichen Gebieten verschärft wird. In den letzten Jahren nahm die Fehlbeanspruchung des Notfalldispositives durch die Bevölkerung zu. Die Notfallstationen der Spitäler berichten über eine hohe Anzahl von «walk-in»-Patienten mit inadäquaten Beschwerden [2, 7]. Kollegen klagen über häufige und unangebrachte Anrufe in ihren Notfalldiensten [8]. Diese Entwicklung wurde auch in anderen Ländern Europas beobachtet. NHS-Direct in England wurde unter anderem aus diesem Grund eingeführt. Auch in Holland und Dänemark waren die Über- und Fehlbeanspruchung des Notfalldispositives mitverantwortlich für tiefgreifende strukturelle und prozessorientierte Reformen in der ambulanten Notfallversorgung. Im Zuge dieser Restrukturierungen wurden die medizinischen Telefondienstleistungen im Notfallbereich ausgebaut und professionalisiert.

\section{Versorgungsplanung und Qualitäts- nachweis des ärztlichen Notfalldienstes}

Bereits heute ist das Telefon ein unverzichtbares technisches Hilfsmittel in jeder Arztpraxis. Tagtäglich werden die Patienten während der Terminvergabe im Rahmen der Sprechstundenplanung von paramedizinischen Pflegefachpersonen medizinisch triagiert. Die Einbindung des Telefons in zeitgemässe Informations- und Kommunikationssysteme erlaubt die Professionalisierung bestehender medizinischer Dienstleistungen. Im Bereiche der ambulanten Notfallversorgung wird dadurch ein wichtiger Schritt zur Umsetzung des im Artikel 58 des Krankenversicherungsgesetzes geforderten Qualitätsnachweises möglich. Eine gegenüber den heutigen Verhältnissen verbessert Datenlage ist eine zentrale Voraussetzung für eine korrekte Versorgungsplanung. Der sinnvolle Einsatz von Informations- und Kommunikationstechnologie ermöglicht eine effiziente, zweckmässige und wirtschaftliche Patientenbetreuung.

Literatur

1 Bunn F, Byrne G, Kendall S. Telephone consultation and triage: effects on the health care use and patient satisfaction. The Cochrane Database of Systematic Reviews 2004. Volume 4.

2 Flaig C, Zehnder K, Zürcher H, Eichenberger P, Frei C, Gegeckas A, et al. Selbsteinweisung ins Spital. PrimaryCare 2002;2:280-3.

3 Meer A, Simonin C, Trapp A, Niemann S, Abel T. Einfluss der medizinischen computerassistierten Telefontriage auf das Patientenverhalten: erste Erfahrungen aus der Schweiz. Schweiz Ärztezeitung 2003;84(41):2160-5.

4 Bovier P, Bouvier Gallacchi M, Goehring C, Künzi B. Wie gesund sind die Hausärzte in der Schweiz? PrimaryCare 2005;5(10):222-8.

5 Adler RH. Emotionale Ursachen von Behandlungsfehlern. Ther Umschau 2005;62(3):157-60.

6 Arnet B, von Below G. Organisation des ambulanten ärztlichen Notfalldienstes in der Schweiz. Schriftenreihe der SGGP 2004. Band 81.

7 Imfeld C. Notfallstation vor dem Kollaps. Pulstipp 2005;Februar:14-6.

8 Grüninger M. Gedanken zum ärztlichen Notfalldienst - eine Zeitbombe? Schweiz Ärztezeitung 2004;85(48):2533. 


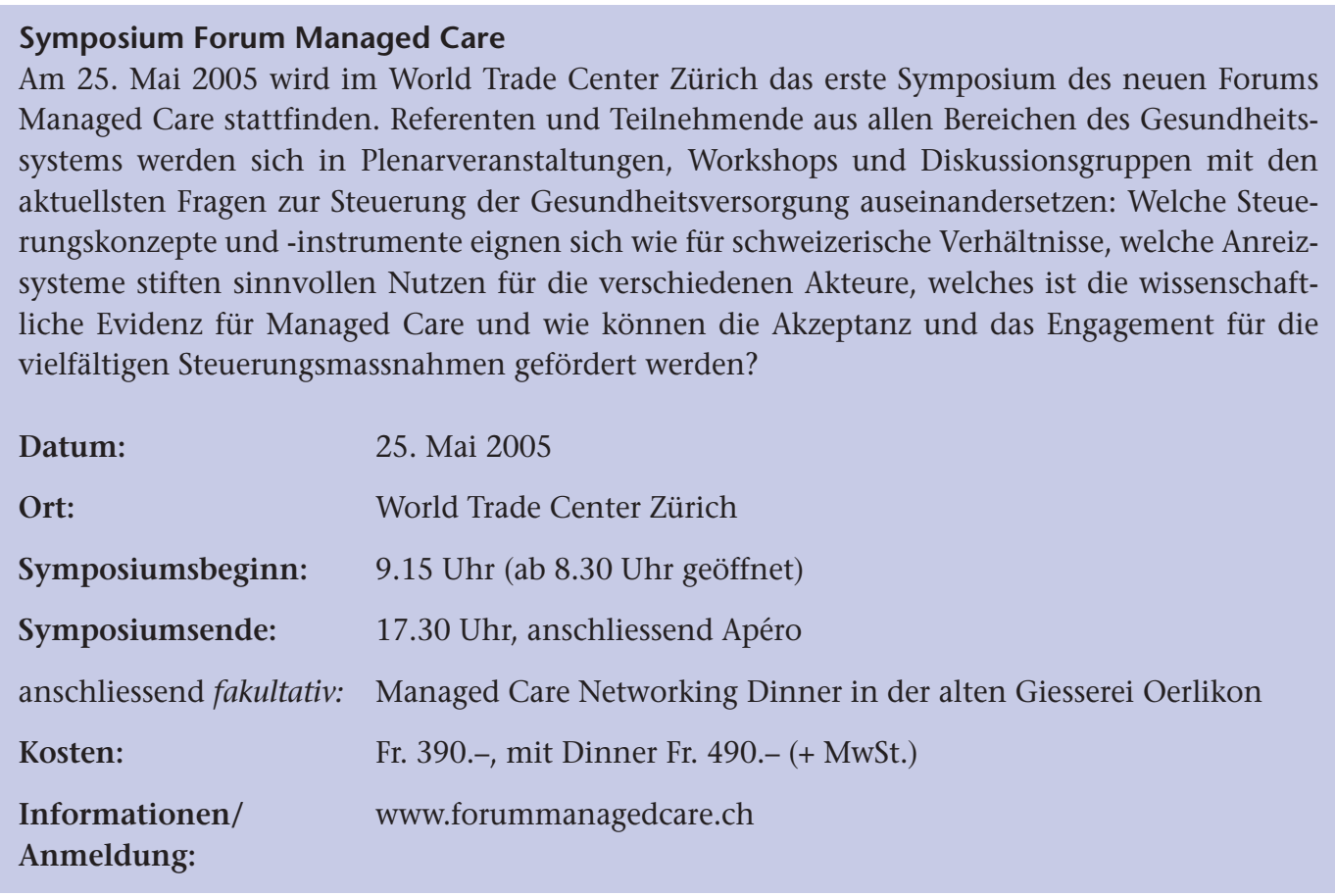

\section{Management- und Führungsseminar für Oberärztinnen und Oberärzte 2005/2006}

zenger \& biaggi

Kurssekretariat: zenger \& biaggi führungsseminare Claudia Baeriswyl Egghölzliweg 4a

Postfach 418

CH-3074 Muri

Tel. 0319517320

Fax 0319517210

E-Mail: jean.biaggi@bluewin.ch
Nachdem sich das Management- und Führungsseminar für Oberärztinnen und Oberärzte bewährt hat, wird es 2005/2006 erneut angeboten und durch Anregungen von Kursteilnehmenden erweitert.

Management- und Führungsaufgaben sind zentrale oberärztliche Aufgaben. Anfänglich steht in der Oberarzttätigkeit wohl der fachliche Aspekt im Vordergrund, gilt es doch, den medizinischen Klinikalltag zu meistern und weitere medizinische Fachkompetenz zu erlangen. In ihrer Tätigkeit werden Oberärztinnen und Oberärzte zunehmend auch mit Managementfragen konfrontiert. Führungskompetenz und soziale Kompetenz werden zu wichtigen Elementen beim Führen der eigenen Abteilungen und in der Vorbereitung der eigenen Karriere.

Das richtige Einsetzen von Managementtools entscheidet weitgehend über Erfolg oder Misserfolg in der täglichen Führungsarbeit. Dar- über hinaus sind Kennen und Verstehen von ausgewählten Grundbegriffen des modernern Managements aber auch unabdingbare Voraussetzungen, um die weitere Zukunft erfolgreich zu gestalten. Dies gilt sowohl für eine zukünftige Karriere als Leitende(r) Ärztin/Arzt oder Chefärztin/-arzt wie auch für eine eigene Praxistätigkeit oder eine alternative Karriere. Management wird oft durch «learning by doing» und durch eigene auch schmerzliche - Erfahrung erlernt. Um die eigenen Erfahrungen strukturiert zu überdenken und weiterzuentwickeln, wurde ein anspruchsvoller Kurs konzipiert, der konzis auf die wesentlichsten Fragen des Führungsalltags eingeht.

Der Kurs wird in Zusammenarbeit mit der FMH und des VSAO angeboten. Er hat die offizielle Anerkennung durch die FMH («FMH approved») erhalten und kann als persönliche Fortbildung anerkannt werden. 


\section{Kursziel}

Ziel ist es, Oberärztinnen und Oberärzten konkrete Erkenntnisse und Tools $\mathrm{zu}$ vermitteln, damit sie ihre eigene Führungs- und Sozialkompetenz reflektieren, optimieren und - situationsgerecht - effizient einsetzen können. Dabei wird gezielt auf die Bedürfnisse der Teilnehmenden eingegangen, sei es im Dialog, in Übungen oder Simulationen. Themenbezogene Referate von Experten geben den theoretischen Hintergrund.

\section{Kursinhalt}

Der Kurs beinhaltet relevante Elemente des Führungs- und Managementalltags von Oberärztinnen und Oberärzten. Die Kursinhalte sind in Zusammenarbeit mit aktiven VSAO-Mitgliedern erarbeitet und auf das Praxisrelevante konzentriert worden.

- Führungsverständnis, Führungsstile und ihre Anwendung, Umgang mit Macht;

- Analyse und Entwicklung des eigenen Führungsstils, Umgang mit Kritik;

- Konfliktanalyse und Konfliktmanagement, Gesetzmässigkeiten und Interventionsmöglichkeiten, Vermeiden von Konflikten;

- Gesprächsführung: Mitarbeitergespräche, Qualifikationsgespräche, schwierige Gespräche (nicht Patientengespräche) usw.;

- Mitarbeiterentwicklung: Mitarbeiter fordern und fördern, Umgang mit schwierigen Mitarbeitern, Teambildung, Vereinbarungskultur;

- Selbstmanagement: persönliches Ressourcenund Zeitmanagement, Balance zwischen Arbeit, Karriere, Familie und Freizeit;

- Projektvorbereitung: Alle Teilnehmenden erhalten die Möglichkeit, eine Thematik aus dem eigenen persönlichen Umfeld im Sinne eines kleinen Projekts weiterzubearbeiten;

- Projektanalyse und Besprechung mit den Kursteilnehmenden und den -verantwortlichen.

\section{Zielpublikum}

- exklusiv Oberärztinnen und Oberärzte;

- mindestens 1 bis 2 Jahre Praxis in Oberarztfunktion;

- offen für alle medizinischen Fachgebiete.

\section{Dozentinnen und Dozenten}

Fachkompetente Experten vermitteln die jeweiligen Kursinhalte theoretisch und praxisbezogen.

\section{Informationen}

\section{Kursdaten}

\begin{tabular}{ll} 
Montag und Dienstag & 3. und 4. Oktober 2005 \\
\hline Donnerstag und Freitag & 8. und 9. Dezember 2005 \\
$\begin{array}{ll}\text { Mittwoch, Donnerstag } & \text { 8., 9. und 10. März 2006 } \\
\text { und Freitag } & \text { 30. Juni 2006 }\end{array}$ \\
\hline $\begin{array}{l}\text { Freitag } \\
\text { M }\end{array}$
\end{tabular}

\section{Kursorte}

Egerkingen und Bern, Tageskurse ohne Übernachtung.

\section{Kurskosten}

8 Kurstage inklusive Mittagessen, Getränke, Pausenverpflegung, Infrastruktur, Kursunterlagen und Projektbetreuung: Fr. 4600.--.

\section{Anmeldeschluss}

31. Juli 2005. Die Teilnehmerzahl ist auf maximal 24 Teilnehmende limitiert. Die Anmeldungen werden nach Eingang berücksichtigt. Im Interesse der Vielfalt der Zusammensetzung werden die Plätze pro Disziplin limitiert.

\section{Kursverantwortliche}

- RA Dr. iur. Christoph Zenger, Bern;

- Dr. med. Jean Biaggi MHA, Muri b. Bern. 